\title{
Unverlässliches Erzählen und romantische Ironie in einem spanischen Roman der Restaurationszeit
}

Narration non fiable et ironie romantique dans un roman espagnol de la Restauration

Unreliable Narration and Romantic Irony in a Spanish Novel of the Restoration

Susanne Greilich

\section{(2) OpenEdition}

Journals

Édition électronique

URL : http://journals.openedition.org/ceg/1450

DOI : $10.4000 /$ ceg. 1450

ISSN : 2605-8359

Éditeur

Presses Universitaires de Provence

Édition imprimée

Date de publication : 15 juin 2015

Pagination : 93-104

ISBN : 978-2-85399-993-9

ISSN : 0751-4239

Référence électronique

Susanne Greilich, « Unverlässliches Erzählen und romantische Ironie in einem spanischen Roman der Restaurationszeit », Cahiers d'Études Germaniques [Online], 68 | 2015, Online erschienen am: 17

Dezember 2017, abgerufen am 02 Dezember 2020. URL : http://journals.openedition.org/ceg/1450 ; DOI : https://doi.org/10.4000/ceg.1450 


\section{Unverlässliches Erzählen und romantische Ironie in einem spanischen Roman der Restaurationszeit}

Susanne GREILICH

Universität Regensburg

\section{Der Erzähler als Lügner - einleitende Bemerkungen zur Theorie der unreliable narration}

Nach der ,Erfindung' des Begriffs der unreliable narration 1961 hat sich Booths Konzept des unzuverlässigen oder auch: unverlässlichen, unglaubwürdigen Erzählens über nahezu 40 Jahre zu einem festen Bestandteil literaturwissenschaftlicher Erzähltheorie entwickelt ${ }^{1}$, bevor es an der Jahrtausendwende eine entscheidende Neuorientierung erfahren hat.

Booth knüpft die Bewertung einer Erzählung als verlässlich oder unverlässlich ${ }^{2}$ an die Idee des implied author. ${ }^{3}$ Die Distanz zwischen implizitem Autor und Erzählinstanz ist damit das entscheidende Kriterium zur Bewertung erzählerischer Verlässlichkeit. Neben der Diskrepanz zwischen den Werte- und Normensystemen des impliziten Autors und des Erzählers, wie Booth sie betont, wurden von der Forschungsliteratur in der Vergangenheit insbesondere kognitive Defizite des Erzählers zur Bestimmung von Unverlässlichkeit zu Grunde gelegt. Demnach meint(e) unreliable narration zwei verschiedene Dinge. Einerseits eine faktische bzw. epistemologische unreliability des Erzählers, die daraus resultiert, dass seine Schilderung nicht den fiktionalen Tatsachen entspricht und andererseits eine moralisch-normative Diskrepanz zwischen Erzähler und implied author, bei der

1 Vgl. entsprechend Ansgar NüNNING, „Unreliable Narration zur Einführung: Grundzüge einer kognitiv-narratologischen Theorie und Analyse unglaubwürdigen Erzählens“, in Ansgar NüNNING, Unreliable narration. Studien zur Theorie und Praxis unglaubwürdigen Erzählens in der englischsprachigen Literatur, Trier, WVT, 1998, S. 3.

2 Vgl. in diesem Zusammenhang die Definition sprachlicher Unverlässlichkeit bei Jahn: ,[...] unverläßlich in sprachlichem Verhalten ist jemand, dessen Diskurs entgegen seinem Anspruch in Darstellung und Urteilsfähigkeit Defizite aufweist und daher nicht als glaubwürdig oder maßgeblich anerkannt werden kann.“ Manfred JAHN, „Package Deals, Exklusionen, Randzonen: das Phänomen der Unverläßlichkeit in den Erzählsituationen“, ibid., S. 82.

3 „I have called a narrator reliable when he speaks for or acts in accordance with the norms of the work (which is to say, the implied author's norms), unreliable when he does not." (Wayne C. Воотн, The Rhetoric of Fiction [1961], Chicago, Chicago UP, 1983, S. 158f.). 
Wertmaßstäbe und ethische Urteile des Erzählers - etwa über das Verhalten der Figuren - als fragwürdig bewertet werden. ${ }^{4}$

Entgegen den terminologischen und theoretischen Problemen, die mit der „undefinierte[n] Verlegenheitsformel“5 ${ }^{\text {"5 }}$ des implied author verbunden sind - wie können etwa die Normen eines impliziten Autors überhaupt ermittelt werden, wie entsteht der Eindruck unverlässlichen Erzählens im Leseprozess - hat Nünning 1998 eine Neukonzeptualisierung der unreliable narration vorgeschlagen. Demzufolge ist der unreliable narrator als ,eine Projektion des Lesers zu verstehen [...], der Widersprüche innerhalb des Textes und zwischen de[r] fiktiven Welt des Textes und seinem eigenen Wirklichkeitsmodell auf diese Weise auflöst" ${ }^{\text {“6 }}$. Damit stellt Nünning die Frage nach textimmanenten Signalen unverlässlichen Erzählens einerseits ebenso wie die nach der Aktivierung außertextlicher Bezugsrahmen (frames of reference) durch den Leser andererseits, greift also auf einen Ansatz kognitiver Theorie zurück. ${ }^{7}$ Die zumeist intuitive Fähigkeit des Lesers, unverlässliches Erzählen als solches zu erkennen, ist damit nicht länger als Ergebnis eines Abgleichs der im Text getroffenen Aussagen mit hypothetischen Annahmen zur , wahren Absicht' des impliziten Autors zu verstehen, sondern vielmehr als Resultat eines Vergleichs textintern gegebener Informationen mit entsprechenden Korrektivinformationen ${ }^{8}$ bzw. eines Vergleichs textinterner Informationen mit den frames of reference der außertextlichen Realität, mithin dem eigenen Erfahrungshorizont des Lesers. ${ }^{9}$

$\mathrm{Zu}$ den textuellen Signalen unverlässlichen Erzählens zählen u.a. explizite Widersprüche des Erzählers bzw. - im Falle polyphoner Texte-kontrastive Versionen desselben Geschehens ${ }^{10}$; autoreferentielle, metanarrative Thematisierungen der eigenen Glaubwürdigkeit; eingestandene Unglaubwürdigkeit, Erinnerungslücken und Parteilichkeit; schließlich häufige Leseransprachen und bewusste Versuche der Rezeptionslenkung durch den Erzähler. ${ }^{11}$

4 Vgl. dazu detaillierter NüNNING, „Einführung“, S. 11. Jahn nimmt im Anschluss an RimmonKenans eine andere Differenzierung vor: „Ein Erzähler ist mimetisch autoritativ, wenn er die Sachverhalte und Ereignisse der Story adäquat (ohne erkennbaren Irrtum oder Irreführung) darstellt; ein Erzähler ist interpretativ autoritativ, wenn er die Sachverhalte und Gegebenheiten der Story in seinen expliziten Kommentaren ohne erkennbare Fehldeutung einschätzt.“(JAHN, „Package Deals“, S. 82-83).

5 NÜNNING, ,Einführung“", S. 13.

6 Ibid., S. 5.

Vgl.ibid., S. 25: „Überblickt man die vorliegende Forschungsliteratur zum Phänomen des unreliable narrator, so kann man sich des Eindrucks nicht erwehren, daß es diese lebensweltlichen kulturellen Modelle und Bezugsrahmen sind, die die Wahrnehmung und Beurteilung der Unglaubwürdigkeit von Erzählinstanzen bestimmen, und nicht allein die vom Text vorgegebenen Informationen. Das bedeutet, daß ein Erzähler nicht an sich unglaubwürdig ,ist', sondern daß es sich dabei um eine Feststellung des Betrachters handelt, die historisch, kulturell und letztlich sogar individuell stark variieren kann.“

8 Vgl. JAHN, „Package Deals“, S. 83.

9 Vgl. NüNNING, „Einführung“, S. 25: „Ob ein Erzähler als unglaubwürdig eingestuft wird oder nicht, hängt somit nicht von der Distanz zwischen seinen Werten und Normen und denen des implied author ab, sondern davon, inwiefern die Weltsicht des Erzählers mit dem Wirklichkeitsmodell des Rezipienten zu vereinbaren ist.““

${ }^{10} \mathrm{Zu}$ den Wirkungszusammenhängen zwischen Polyphonie und unverlässlichem Erzählen vgl. Felicitas Menhard, Conflicting Reports. Multiperspektivität und unzuverlässiges Erzählen im englischsprachigen Roman seit 1800, Trier, WVT, 2009.

11 Vgl. NÜNNING, „Einführung“, S. 27f.; Dagmar BuscH, „Unreliable Narration aus narratologischer 
In der Aufzählung einschlägiger Typen, in die sich - Jahn zufolge - unverlässliche Figuren einreihen lassen oder besser: mit denen sich unverlässliche Erzähler mit Hilfe eines lebensweltlichen Bezugs typologisieren lassen - etwa als Heuchler, Angeber, Aufschneider, Träumer, Naive, Engstirnige, Besessene, Unverbesserliche, Irre, Verwirrte, Ignoranten, Blender, Verblendete, Neurotiker usw. ${ }^{12}$ - finden sich auch jene Typen, die im Kontext dieses Bandes von besonderem Interesse sind: der Lügner und der Täuscher. Am Beispiel eines spanischen Romans aus dem späten 19. Jahrhundert, nämlich Valeras Pepita Jiménez (1874), werden im nun Folgenden die Formen erzählerischer Lüge beleuchtet werden. Abschließend wird umrissen, welche Funktion dem Gebrauch unverlässlichen Erzählens bei Valera im Kontext der spanischen Literatur der Restaurationszeit ${ }^{13}$ und ihrer poetologischen Debatten zukam, um damit einen ersten Schritt in Richtung der Beseitigung eines Forschungsdesiderats zu gehen, das Nünning in seinem programmatischen Aufsatz formuliert hat. ${ }^{14}$

\section{Nichts als Lügner? - Juan Valeras Pepita Jiménez (1874)}

Pepita Jiménez, der bekannteste Roman Juan Valeras, erzählt die Geschichte des Priesteranwärters Luis de Vargas, der in seinem andalusischen Heimatstädtchen die junge, von seinem Vater Don Pedro umworbene Witwe Pepita kennenlernt. Zunächst voller Vorurteile gegenüber der schönen, klugen und selbstbewussten Frau gerät Luis zunehmend in ihren Bann, bis er schließlich in einem ausweglos erscheinenden Konflikt zwischen religiöser Berufung und weltlicher Liebe gefangen ist. Luis' Begehren, das er sich selbst nur zögerlich eingesteht, kulminiert in einer Liebesnacht mit Pepita. In einem Duell mit einem Widersacher verwundet, gesteht Luis seinem Vater auf dem Krankenbett alles. Don Pedro verzichtet auf seine Ansprüche, die jungen Leute heiraten und beginnen - so erfährt der Leser am Schluss - eine vorbildliche Ehe zu führen.

Augenfällige strukturelle Merkmale des vierteiligen Romans - der sich aus einem Prolog, den „Cartas de mi sobrino“ („Briefe meines Neffen“), einem „Paralipómenos“ („Paralipomena“) betitelten Teil und einem Epilog („Cartas de mi hermano“, dt.: „Briefe meines Bruders“) zusammensetzt - sind die für Beginn

Sicht: Bausteine für ein erzähltheoretisches Analyseraster“, in NüNNING, Unreliable narration, S. 41-58; Gaby Allrath, „,But why will you say that I am mad?“ Textuelle Signale für die Ermittlung von unreliable narration“, ibid., S. 59-79.

12 Vgl. JAHN, ,Package Deals“, S. 82.

13 Die spanische Restauration, Epoche der Wiederherstellung der Monarchie, umfasst in der weiteren Begriffsbestimmung die Zeitspanne zwischen der Ersten und der Zweiten Spanischen Republik, also die Jahre 1874/75-1931. Im engeren Sinne wird unter der Restauration der Zeitraum von 1874/75 bis zum desastre 1898 verstanden, als Spanien seine letzten Kolonialgebiete verlor. Vgl. José María JovER ZAMORA, „Restauración y conciencia histórica“, in Real Academia de la Historia, España: Reflexiones sobre el ser de España, Madrid, Real Academia de la Historia, 1998, S. 331.

14 Vgl. dazu NüNNING, ,Einführung“, S. 36: „Völlig ungeklärt sind etwa die historisch und kulturell variablen Funktionen, die der Gebrauch von unreliable narration erfüllen kann, sowie die dialogische Beziehung dieser Erzählform zu anderen Diskursen.“ 
und Ende gewählte Briefform und die Polyphonie. Der Roman hat fünf Erzähler. Unter diesen kommt dem Herausgeber des allograph fiktiven Vorworts ${ }^{15}$, das der eigentlichen Romanhandlung vorangestellt ist, eine besondere Funktion zu: Er kommentiert das Konvolut, bewertet die Urheberschaft und schaltet sich auch im weiteren Romanverlauf immer wieder ein. Die editorialen Kommentare fungieren als Rahmung der Geschichte, der fiktive Herausgeber wirkt als übergeordnete Instanz. ${ }^{16}$ Die Teile I und III: „Cartas de mi sobrino“ und „Epílogo - Cartas de mi hermano" bedienen sich der Ich-Erzähler Luis bzw. Pedro de Vargas. Für Teil II, die Paralipomena, wird der Onkel des Protagonisten, ein Dechant, als Urheber genannt. Ergänzende Informationen über das Verhältnis zwischen Pepita und Luis bilden den Kern dieses mittleren Textteils, in dem die Liebenden ausführlich zu Wort kommen und sich ebenfalls ein Erzähler angelegentlich einschaltet. ${ }^{17}$ Insbesondere werden Auslassungen des Berichts kommentiert, der neben dem Gebot der Kürze dem Prinzip der ,Wahrheit' unterworfen wird: „Wir mögen Pepita sehr, doch die Wahrheit steht über allem und so müssen wir es sagen, auch wenn es das Ansehen unserer Heldin beschädigt ““ ${ }^{18}$ heißt es im Text. ${ }^{19}$

Diese Einlassung des Erzählers ist als wichtiges Signal in Richtung des Lesers zu werten, denn tatsächlich ist ein zentrales Motiv des Romans das Ringen um die narrative Wahrhaftigkeit des Erzählten, an dessen Prozess alle Erzähler beteiligt sind. Den Beginn macht im Prolog zu Pepita Jiménez der fiktive Herausgeber. Mit dem Herausgebervorwort ruft Valera eine Gattungstradition des Briefromans auf, die sich in Frankreich seit Beginn des 18. Jahrhunderts herausbildete und die zugleich ihre Wurzeln im spanischen Ritterroman und bei Cervantes hat. ${ }^{20}$ Die

15 Vgl. Gérard Genette, Seuils, Paris, Éd. du Seuil, 1987, S. 175. In der von Genette so genannten „préface allographe fictive“ ist die Urheberinstanz des Vorworts ebenso fiktiv wie die Erzählinstanzen der eigentlichen Haupterzählung, jedoch nicht mit diesen identisch.

${ }^{16}$ Zur Rahmungsfunktion der Herausgeberfunktion und den Unterschieden zwischen bzw. Gemeinsamkeiten von Rahmenerzählung und Herausgeberfiktion vgl. Uwe WIRTH, Die Geburt des Autors aus dem Geist der Herausgeberfiktion. Editoriale Rahmung im Roman um 1800: Wieland, Goethe, Brentano, Jean Paul und E.T.A. Hoffmann, München, Wilhelm Fink, 2008, S. 160-164.

17 So situiert er zu Beginn der Paralipomena die Erzählung in zeitlicher Hinsicht („A los cinco días de la fecha de la última carta que hemos leído empieza nuestra narración“, S. 249; Dt.: „Fünf Tage nach dem letzten Brief, den Don Luis seinem Onkel und geistlichen Berater geschrieben, hebt die Fortsetzung der Erzählung an [...]“", S. 150), kommentiert die Gefühle Don Luis’ für Pepita (,No se crea, con todo, que no amaba a la joven viuda.“, S. 264; Dt.: „Nicht als ob er die junge Frau nicht liebte.“, S. 173 ), rechtfertigt den Sprachgebrauch des Conde de Genazahar in seiner Unterhaltung mit Don Luis über Pepita sowie den Antoñonas im Gespräch mit Luis de Vargas. Sämtliche spanische Textzitate nach der Ausgabe Juan Valera, Pepita Jiménez, hrsg. v. Leonardo Romero, Madrid, Cátedra, 2011. Deutsche Übersetzung der Zitate - sofern nicht anders angegeben - gemäß der Ausgabe: Juan VALERA, Pepita Jiménez. Roman. Aus dem Spanischen von Annemarie und Fritz WAHL, Zürich, Manesse-Verl., 1950.

18 Übersetzung von mir. Die Textausgabe von Wahl unterschlägt diese Passage des spanischen Originals: „Mucho queremos a Pepita; pero la verdad es antes que todo, y la hemos de decir aunque perjudique a nuestra heroína“" (VAlera, Pepita Jiménez, S. 294).

${ }^{19}$ Integriert ist ferner ein Briefwechsel zwischen dem Dechanten und Pedro de Vargas, der von letzterem verlesen wird (ibid., S. 337ff.). In vertikaler Vervielfältigung der Stimmen macht der (auktoriale) Erzähler der Paralipomena die Figur Don Pedro selbst zum Erzähler einer sich zwischen zwei Figuren entspinnenden Korrespondenz.

${ }^{20}$ Romero bemerkt ergänzend dazu, dass die Referenz auf Quellen der außertextlichen Realität auch in der spanischen Romantik ein Topos war, wobei die Romantiker auf mündliche Quellen, nicht auf 
Beschreibung des Textursprungs (ein durch Zufall in die Hände des Herausgebers gelangtes Manuskript eines verstorbenen Bekannten), die scheinbar rücksichtsvolle Verschleierung der vollen Identität des Verfassers und der im Text auftretenden Personen, die Versicherung der Authentizität des Dokuments, die Beschreibung des ursprünglichen Manuskripts und der wenigen daran vorgenommenen Veränderungen: all dies sind typische Elemente fingierter Herausgeberschaft.

Zum besseren Verständnis sei an die verschiedenen Formen des Vorworts erinnert, wie sie Genette in der ihm eigenen Systematik unterschieden hat. ${ }^{21}$ Zentraler Ausgangspunkt ist die instance préfacielle, d.h. die Frage danach, 1. wem die Urheberschaft des Vorworts als para- bzw. peritextuellem Element zugeschrieben wird: einem Autor (auctorial), einer Figur der Diegese (actorial) oder einer anderen, dritten Person (allographe) und ob 2. diese Urheberschaft als authentisch (authentique), unecht (apocryphe) oder fiktiv (fictif) wahrgenommen wird. ${ }^{22}$ Zusätzlich untersucht Genette die Äußerung der Vorwortinstanz in Hinblick auf den Text der Haupterzählung. Verneint die Vorwortinstanz die Urheberschaft dieses Textes, so ist der Prolog als verneinend auktoriales Vorwort ${ }^{23} \mathrm{zu}$ charakterisieren. Das Resultat dieses Verfahrens ist in der Tat aber eine fingierte Herausgeberschaft: Der Autor tut so, als ob er nur als Herausgeber eines Dokuments fungierte, während er tatsächlich Urheber des gesamten fiktionalen Textes ist. In diesem Sinne verwendet auch die deutsche Forschung den Begriff des fingierten Herausgebers ${ }^{24}$ bzw. die spanische Forschung den Begriff des apócrifo - und in diesem Sinne wird er auch hier verwendet werden.

Das Verfahren, im Romanvorwort die Verantwortung für die Herausgabe der ,Dokumente' zu übernehmen, ihre Urheberschaft aber einem oder mehreren, vorzugsweise anonymisierten Verfassern zuzuschreiben, stellte zunächst ein ästhetisches Mittel dar, sich u.a. dem häufig an den Roman gerichteten Vorwurf der Lüge zu entziehen ${ }^{25}$, indem Authentizität im Sinne einer objektiven, referentiellen Wahrheit behauptet wurde. Der Roman bediente sich mit der fingierten Herausgeberschaft also einer Lüge, um Wahrheit zu reklamieren. Im Verlauf des 18. Jahrhunderts verschob sich der Fokus zunehmend in Richtung einer die interne, narrative Wahrhaftigkeit des Romans thematisierenden Diskussion. ${ }^{26}$ Zugleich wurde die fingierte Herausgeberschaft zum Anlass einer Metareflexion über die Kategorien von Fiktion und Realität, für die das Romanvorwort der privilegierte Ort war.

\footnotetext{
Manuskripte, verwiesen (vgl. ibid., S. 136).

21 Vgl. Genette, Seuils, S. 166-180.

${ }^{22}$ Aus der Kreuzung der genannten Kategorien ergeben sich insgesamt 9 Möglichkeiten, die Vorwortinstanz bzw. das Vorwort zu beschreiben (vgl. ibid., S. 169).

23 ,instance préfacielle auctoriale authentique dénégative“" (ibid., S. 172).

24 Vgl. etwa WirTh, Geburt des Autors, S. 146.

${ }^{25}$ Im französischen 18. Jahrhundert war der Begriff des roman in genereller Form als Bezeichnung für einen ebenso unwahren wie unwahrscheinlichen Bericht geläufig, weshalb sich u.a. die noch vielfach auf eine unsichere Quellen- und Informationslage fußenden Gazetten den Vorwurf gefallen lassen mussten, nichts weiter als romans hebdomadaires zu sein. Vgl. dazu etwa Yannick SEITÉ, „Le ,Roman Hebdomadaire", fiction et information dans la gazette au XVIII ${ }^{\mathrm{e}}$ siècle", in Malcolm Cook/ Annie Jourdan, Journalisme et fiction au $18^{e}$ siècle, Bern, Peter Lang, 1999, S. 63-74.

${ }^{26}$ Vgl. Jan Herman, Le Mensonge romanesque. Paramètres pour l'étude du roman épistolaire en France, Amsterdam, Rodopi, 1989 und Jacques Rustin, „Mensonge et vérité dans le roman français du XVIIIle siècle“, in Revue d'Histoire Littéraire de la France, 1, 1969, S. 13-38.
} 
Quoique je ne porte ici que le titre d'éditeur, j'ai travaillé moi-même à ce livre, et je ne m'en cache pas. Ai-je fait le tout, et la correspondance entière est-elle une fiction? Gens du monde, que vous importe? C'est sûrement une fiction pour vous ${ }^{27} \ldots$

So leitet Rousseau die Julie ein. Indem sich bei der Leserschaft allmählich die Erkenntnis durchsetzte, dass der Autor zugleich Verfasser des Vorworts wie auch des eigentlichen Romantextes war, und gleichzeitig die Bekundung des Gegenteils im Prolog beibehalten wurde, konnte sich der spielerische Charakter der fingierten Herausgeberschaft zunehmend entfalten, wie ihn auch Genette als charakteristisch für alle fiktionalen Vorworte annimmt.

Dabei verlor gleichzeitig der tatsächliche Akt des Fingierens seine eigentliche Wirkung. Der Sprechakttheoretiker Searle unterscheidet zwischen dem Fingieren als Vorgeben mit Täuschungsabsicht und der Fiktion als Vorgeben ohne Täuschungsabsicht ${ }^{28}$ und greift damit eine Unterscheidung auf, wie sie bereits Hamburger in Logik der Dichtung vorgenommen hatte. ${ }^{29}$ Ausgehend von der „Seinsweise dessen, was nicht wirklich ist ${ }^{\star 630}$ ist das Fiktive „wahr, weil es zugibt, nicht wirklich zu sein ${ }^{\text {“31 }}$. Diese von der Autorenseite her gedachte Unterscheidung ist von Wirth $2008 \mathrm{im}$ Anschluss an Austins Untersuchung zum Sprechakt des pretending (Vorgeben, illukotionär) und des making believe (Glauben machen, perlokutionär) in Richtung der Rezipientenseite gewendet worden: „es geht nicht mehr darum, ob der Autor beim fiktionalen So-Tun-Als-Ob Täuschungsabsichten hat, sondern umgekehrt darum, ob der Rezipient die Täuschungsversuche ernst nimmt oder nicht". ${ }^{32}$ Das Herausgebervorwort in Pepita Jiménez konnte vom Leser des ausgehenden 19. Jahrhunderts unmittelbar als Gattungselement und Bestandteil der literarischen Fiktion erkannt werden, der Herausgeber selbst als Figur der erzählten Welt. ${ }^{33}$

\footnotetext{
${ }^{27}$ Jean-Jacques Rousseau, Julie ou La Nouvelle Héloïse, hrsg. v. Michel LaunAY, Paris, GarnierFlammarion, 1967, S. 3.

${ }^{28}$ Vgl. John R. Searle, „Der logische Status fiktionalen Diskurses“, in John R. Searle, Ausdruck und Bedeutung. Untersuchungen zur Sprechakttheorie [1979], Frankfurt a. M., Suhrkamp, 1982, S. 87.

${ }^{29}$ Hamburger unterscheidet zwischen dem Fiktiven und dem Fingierten und macht diese Unterscheidung im Besonderen an der „Bewusstseinshaltung“ fest: „Der Begriff des Fingierten bedeutet ein Vorgegebenes, Uneigentliches, Imitiertes, Unechtes, der des Fiktiven dagegen die Seinsweise dessen, was nicht wirklich ist: der Illusion, des Scheins, des Traums, des Spiels“ (Käte Hamburger, Die Logik der Dichtung, Stuttgart, Klett, 1957, S. 247). Vgl. zu dieser Thematik detaillierter WIRTH, Geburt des Autors, S. 122-126.

${ }^{30}$ Hamburger, Logik der Dichtung, S. 247.

31 WIRTH, Geburt des Autors, S. 123.

${ }^{32}$ Ibid., S. 126.

${ }^{33}$ Ansorge betont in seiner Studie zu den Arten der Vorrede im Briefroman mit Blick auf den Herausgeber: „Die Herausgeber-Rolle [d.i. die instance préfacielle auctoriale authentique dénégative in der Terminologie Genettes, Ergänzung von mir] betrachten wir als unecht, die Herausgeber-Figur [d.i. die instance préfacielle fictive nach Genette, Ergänzung von mir] als gedichtet und damit als echt in einer nicht-wirklichen Welt, nämlich der des Romans." (Hans-Jürgen Ansorge, Art und Funktion der Vorrede im Roman. Von der Mitte des 18. Jahrhunderts bis zum Ende des 19. Jahrhunderts, Diss., Würzburg, 1969, S. 75).
} 
Diese Feststellung schafft nun aber das Problem unverlässlichen Erzählens nicht aus der Welt, ja man könnte sogar behaupten, dass es sich im Kontext des fiktiven Herausgebers erst eigentlich stellt. Denn wo die fingierte Herausgeberschaft Anlass bietet zur spielerischen Metareflexion über die Kategorien von Realität und Fiktion, da stehen der fiktionale Charakter weder des Vorworts noch des restlichen Romantextes in Pepita Jiménez noch in Frage. Steht das fingierte Herausgebervorwort bzw. die Herausgeberrolle, derer sich der Romanautor im Vorwort bedient, in der Tat noch an der „Schwelle“ zwischen lebensweltlicher Realität und literarischer Fiktion ${ }^{34}$, so haben sich der fiktive Herausgeber und sein Vorwort von den ,Texträndern“ in die erzählte Welt selbst, vom ,dehors“ ins „dedans“ ${ }^{\text {“35 }}$ bewegt. ${ }^{36}$ Als Teil der Gesamtfiktion unterliegt das Vorwort, mithin die Rede des Herausgebers als Erzähler mit allen darin enthaltenen Äußerungen, nun vielmehr der Frage nach narrativer Zuverlässigkeit und Glaubwürdigkeit.

In Pepita Jiménez erweist sich der fiktive Herausgeber nun tatsächlich als unverlässliche Erzählinstanz. Die anfängliche Versicherung, das Manuskript unverändert in den Druck gegeben $\mathrm{zu}$ haben $^{37}$, wird im weiteren Romanverlauf konterkariert, als der Herausgeber unumwunden eingesteht, an den Paralipomena umfassendere Modifikationen vorgenommen zu haben: Mit Rücksicht auf die Gegebenheiten des zeitgenössischen Marktes habe er die Glossen des Dechanten gestrichen. ${ }^{38}$ Ganz offensichtlich hat der Herausgeber in Hinblick auf seinen Anteil an der Textproduktion zunächst gelogen, ist er nicht nur Editor, sondern beinahe Co-Autor der novela. Dabei stellt sich die Frage, inwiefern dieses Eingeständnis für den Leser überraschend kommt. Mit dem Geständnis der Herausgeberfigur spiegelt Valera nämlich im Grunde nur ein Element fingierter Herausgeberschaft, in der das

${ }^{34}$ Genette spezifiziert: „Le paratexte est donc pour nous ce par quoi un texte se fait livre et se propose comme tel à ses lecteurs, et plus généralement au public. Plus que d'une limite ou d'une frontière étanche, il s'agit ici d'un seuil, ou - mot de Borges à propos d'une préface - d'un 'vestibule' qui offre à tout un chacun la possibilité d'entrer, ou de rebrousser chemin. 'Zone indécise' entre le dedans et le dehors, ellemême sans limite rigoureuse, ni vers l'intérieur (le texte) ni vers l'extérieur (le discours du monde sur le texte), lisière ou, comme disait Philippe Lejeune, 'frange du texte imprimé qui, en réalité, commande toute la lecture" " (GenetTe, Seuils, S. 7f.).

35 Ibid., S. 8.

${ }^{36}$ Insbesondere im Falle der fiktiven Herausgeberschaft ist das Vorwort im Grunde nicht mehr als Teil des Paratextes, sondern als integraler Bestandteil der Erzählung selbst zu betrachten. Vgl. in diesem Zusammenhang Werner WoLF, Ästhetische Illusion und Illusionsbrechung in der Erzählkunst. Theorie und Geschichte mit Schwerpunkt auf englischem illusionsstörendem Erzählen, Tübingen, Niemeyer, 1993, S. 222.

37 „fielmente trasladado a la estampa“, „mudando sólo los nombres propios“ (VALERA, Pepita Jiménez, S. 137). Dt.: „Und nun folgt buchstabengetreu das erwähnte Manuskript“, „Nur die Namen hab ich geändert [...]“ (Valera, Pepita Jiménez. Roman, S. 7 und S. 6).

38 „Lo que sí hizo fue poner glosas y comentarios de provechosa edificación, cuando tal o cual pasaje lo requería; pero yo los suprimo aquí, porque no están en moda las novelas anotadas o glosadas, y porque sería voluminosa esta obrilla si se imprimiese con los mencionados requisitos“" (VALERA, Pepita Jiménez, S. 321). Dt.: „Was der Herr Dekan dann doch tat, war, die ein oder andere Passage, wo es ihm angebracht erschien, mit erbaulichen Glossen und Kommentaren zu versehen, doch ich streiche sie hier allesamt, da annotierte und kommentierte Texte nicht in Mode sind und dies kleine Werk einen erheblichen Umfang erhalten würde, wenn man es unter den genannten Voraussetzungen drucken wollte." [Übersetzung von mir. Die deutsche Fassung des Textes von Wahl weicht an dieser Stelle deutlich vom spanischen Original ab und verfälscht die eigentliche Aussage]. 
Eingeständnis partieller Autorschaft zunehmend zur Konvention geworden war. Konnte der Leser des 19. Jahrhunderts von der Herausgeberfigur des Romans etwas anderes erwarten als dieses Eingeständnis, gelogen zu haben?

Auch Luis de Vargas als Ich-Erzähler der „Cartas de mi sobrino“ “ist als unverlässlich einzustufen, versucht er doch über lange Strecken des Romans, sich selbst, den Dechanten und damit auch den Leser über seine wahren Gefühle für Pepita zu täuschen. Aus der Diskrepanz zwischen Luis' Versicherungen über sein Desinteresse an Pepita und widersprüchlichen Textsignalen (insbesondere etwa die Vielzahl der Stellen, an der er auf die junge Witwe zu sprechen kommt) resultiert ein Großteil des humorigen Charakters, jener ,pleasing ambiguity“393, die dem Roman zu eigen ist. Bereits die lateinische Sentenz „Nescit labi virtus“ (,Die Tugend kann nicht fallen“/ „Die Tugend weiß nichts vom Fallen“), die als Maxime den Roman überschreibt, gibt dem Leser einen Hinweis darauf, dass Luis' moralische Standfestigkeit einer schweren Prüfung unterzogen werden wird. Dieser Informationsvorsprung des Lesers und die daraus resultierende Diskrepanz zwischen der Absicht des Erzählers (nämlich glaubhaft zu versichern, dass er sich überhaupt nicht für Pepita interessiere) und dem Wissensstand des Lesers hat zur Folge, dass der Romantext von Beginn an mit dem Vorzeichen der dramatischen Ironie versehen ist: Mit Chatman können wir das unverlässliche Erzählen als Form dramatischer Ironie begreifen..$^{40}$

Schließlich erscheint auch der Paralipomena-Erzähler als unglaubwürdige Instanz. Wird der Erzähler, respektive Verfasser der Paralipomena von der Herausgeberfigur zunächst als absolut verlässlich qualifiziert ${ }^{41}$ und seine Glaubwürdigkeit durch den Verweis auf Berufsstand (Kirchenmann) und das Verwandtschaftsverhältnis zum Protagonisten (Onkel) über jeden Zweifel erhoben, so wird diese Einschätzung im weiteren Textverlauf sukzessive aufgeweicht. Dies geschieht über einen zweifachen Erzählerkommentar des Dechanten selbst. ${ }^{42}$ Beide Male zielt der Kommentar augenscheinlich darauf ab, dem Eindruck von Unverlässlichkeit vorzubeugen, den die Geschichte durch die Wortwahl der Figuren beim Leser auszulösen Gefahr läuft, erzielt dadurch aber den genau gegenteiligen Effekt: „Ernsthafte Leute, die nicht in derartigen Herrenklubs verkehren, werden sich von solch brutaler und maßloser Sprache, [die nahezu unwahrscheinlich erscheint, Ergänzung von mir], angewidert fühlen. Aber andere, die sich in dem Milieu häufiger bewegen, müssen bestätigen, daß die feinsten Damen [...] in diesen Zirkeln oft Zielscheibe eines infamen und schmutzigen Spotts werden “43, heißt es in Hinblick auf die Unterhaltung von Don Luis

${ }^{39}$ Robert E. Lotт, ,'Pepita Jiménez' and 'Don Quixote': A structural comparison“, in Hispania, 45 (1), 1962, S. 395.

${ }^{40}$ Vgl. Seymour Benjamin Chatman, Story and discourse. Narrative structure in fiction and film, Ithaca, Cornell University Press, 1978.

${ }^{41}$ „un sujeto, perfectamente enterado de todo“ (VALERA, Pepita Jiménez, S. 247). Dt.: ,jemand, der den Vorgängen in allen Einzelheiten nahe war" (VALERA, Pepita Jiménez. Roman, S. 146).

${ }^{42}$ Es bleibt letzten Endes unklar, ob es tatsächlich der Dechant ist, der hier als Erzähler zu Wort kommt. Angesichts des Spiels mit der Identität des Paralipómenon-Erzählers ist es auch möglich anzunehmen, der Herausgeber komme hier mit einem satirisch gefärbten Kommentar zu Wort.

43 Valera, Pepita Jiménez. Roman, S. 182. „[...] [L]es parecerá desbocado y brutal hasta la inverosimilitud; pero los que conocen el mundo confesarán que este lenguaje es muy usado en él [...]“ (Valera, Pepita Jiménez, S. 272). 
mit dem Conde de Genazahar, bevor der Erzähler kurz darauf den Sprachstil der Dienerin Antoñona vor dem Vorwurf der inverosimilitud in Schutz nimmt:

[...] su lenguaje fue tan digno y urbano, que no faltaría quién le calificáse de apócrifo, si no se supiese con la mayor evidencia todo esto que aquí se refiere, y si no constasen, además, los prodigios de que es capaz el ingénito despejo de la mujer, cuando le sirve de estímulo un interés o una pasión grande. ${ }^{44}$

Die übertriebene Versicherung der,Echtheit' des Erzählten im zweiten Textbeispiel und die Referenz auf eine außertextliche Wirklichkeit im ersten verweisen dabei umso deutlicher auf die Unglaubwürdigkeit (,apócrifo“, ,inverosimilitud“) der Erzählung, wie auch auf ihre ästhetische Unzulänglichkeit. Die offensichtliche Diskrepanz zwischen dem ,unnatürlichen“ Sprachgebrauch Antoñonas und der Versicherung des Erzählers, er berichte streng und allein nach seinem Wissen, lassen Zweifel an der Zuverlässigkeit der Quelle und der vom Erzähler versicherten Zeugenfunktion aufkommen, die durch den in seiner Abgeschmacktheit nahezu hilflos anmutenden Erklärungsansatz für die überraschende Eloquenz Antoñonas in der zweiten Parataxe (die Wunder des weiblichen Verstandes) zusätzlich genährt werden.

Der Eindruck unglaubwürdigen Erzählens entsteht durch den expliziten Appell des Erzählers an den Erfahrungshorizont des Lesers. Indem dieser die Einlassungen Antoñonas mit seinem frame of reference abgleicht, kann er gar nicht anders, als den Erzähler als unverlässlich zu qualifizieren. Diesem Urteil unterliegt die Bewertung erzählerischer Glaubwürdigkeit im weiteren Textverlauf. Dabei erscheint der Erzähler zunehmend weniger als neutraler Berichterstatter der Ereignisse, als der er zunächst qualifiziert worden war, denn als engagierter Autor einer Geschichte, in der er seine eigenen moralischen Werte und sprachlichen Duktus den Figuren in den Mund legt. Insbesondere die Selbstbezichtigungen Pepitas, sie sei eine „höllische Sünderin“ und eine „Götzendienerin“ lassen dies zu Tage treten. ${ }^{45}$ Dabei ist die

44 Valera, Pepita Jiménez, S. 280. Dt.: „Man kann nicht leugnen, daß Antoñona sich in diesem Fall sehr umsichtig benahm und sich einer durchaus würdigen und höflichen, ja sogar bewundernswerten Sprache bediente. [Es fehlte gewiss nicht an Jenen, die ihre Sprache als zweifelhaft erachteten, wenn man nicht mit völliger Gewissheit all dies wüsste, was hier berichtet wird, Ergänzung von mir] [...] was bringt der aufgeweckte Verstand einer Frau nicht alles zustande, sobald ihn ein wirkliches Interesse oder gar eine Leidenschaft bewegt!“ (Valera, Pepita Jiménez. Roman, S. 195).

45 Vgl. Valera, Pepita Jiménez, S. 309: „Ahora conozco cuán vil es el metal del que estoy forjada y cuán indigno de que le penetre y mude el fuego divino. Lo declararé todo, desechando hasta la vergüenza. Soy una pecadora infernal. Mi espíritu grosero e inculto no alcanza esas sutilezas, esas distinciones, esos refinamientos de amor.“; sowie ibid., S. 310: „Yo amo en usted, no ya sólo el alma, sino el cuerpo, y la sombra del cuerpo en los espejos y en el agua, y el nombre y el apellido, y la sangre, y todo aquello que le determina como tal don Luis de Vargas; el meta de la voz, el gesto, el modo de andar y no sé qué más diga. Repito que es menester matarme. Máteme usted sin compasión, No: yo no soy cristiana, sino idólatra materialista."

Dt.: „Jetzt erkenne ich, wie häßlich das Metall sein muß, aus dem ich geschmiedet. Ich bin wahrlich unwürdig, daß das göttliche Feuer mich läutere und durchdringe. Ich werde Ihnen alles ohne Scham bis aufs letzte bekennen. Ich bin eine höllische Sünderin! Mein gemeiner und simpler Geist begreift solche Feinheiten, solche Unterschiede, ein solches Raffinement der Liebe nicht.“ (VAlERA, Pepita Jiménez. Roman, S. 236) und weiter: „Ich liebe nicht nur ihre Seele, sondern Ihren Körper und dessen Widerschein im Spiegel, wie im ruhenden Wasser, Ihren Vor- und Nachnamen, Ihr Blut und alles, was den Don Luis de Vargas nun einmal ausmacht. Ich liebe den Klang Ihrer Stimme, die Gebärde, die Art, wie Sie schreiten, und ich weiß nicht, was noch. Ich muß es Ihnen noch einmal wiederholen, bringen Sie mich um, ermorden 
Selbstanklage der jungen Witwe als verfälschte Darstellung der Erzählerfigur zu qualifizieren. Dieser Verdacht wird genährt durch die offensichtliche Diskrepanz zwischen der Darstellung Pepitas in den Briefen Luis de Vargas und der Version des Verhältnisses der Beiden, wie sie die Paralipomena präsentieren: Bei dem einen mariengleiche Unschuld ist sie bei dem anderen die lockende Sünde in Person. Es liegt die Vermutung nahe, dass der Dechant hier unzuverlässig erzählt, um den Verzicht auf das Priesteramt des von ihm über Jahre erzogenen und ausgebildeten Neffen weniger als moralische Schwäche des jungen Mannes, denn als Resultat weiblicher Untaten erscheinen zu lassen.

In Pepita Jiménez werden also verschiedene Dynamiken wirksam. Der Verdacht der Unverlässlichkeit des bzw. der Erzähler entwickelt sich zum einen unabhängig von der Polyphonie des Textes: ${ }^{46}$ Der Herausgeber als Erzähler des Vorworts entlarvt sich im Verlauf des Romans durch eigenen Kommentar als unverlässliche Instanz, die Unverlässlichkeit des Ich-Erzählers Luis de Vargas in Hinblick auf seine Gefühle für Pepita wird innerhalb der Erzählung von Luis' selbst durch widersprüchliche Textsignale (wie auch durch die Interaktion zwischen Textinformation und Weltwissen des Lesers) deutlich. Auch der Erzähler der Paralipomena löst zunächst durch eigenen Verweis auf die Erfahrungswelt der Leser jenen Rezeptions- und Deutungsprozess aus, an dessen Ende der Befund der unreliable narration steht ein schönes Beispiel für den in der Literatur bisher eher selten behandelten Fall unverlässlichen Erzählens bei auktorialer Erzählsituation. ${ }^{47}$ Zum anderen wird der Verdacht der unreliable narration über die diskrepanten Darstellungen Pepitas ausgelöst, die der Polyphonie des Textes geschuldet sind.

Der Zweifel an der Verlässlichkeit der Erzählinstanzen ist dabei zu guter Letzt auch an die Frage nach der Identität des Paralipomena-Erzählers geknüpft.

Dije al empezar que me inclinaba a creer que esta parte narrativa o Paralipómenos era obra del señor Deán [...] pero [...] al notar la libertad con que se tratan ciertas materias y la manga ancha que tiene el autor para algunos deslices, dudo que el señor Deán, cuya rigidez sé de buena tinta, haya gastado la de su tintero en escribir lo que el lector habrá leído. $^{48}$

Mit diesem Kommentar sät der Herausgeber gegen Ende von Teil III nicht nur Zweifel an der Autorschaft der Paralipomena, vielmehr entlarvt er sich selbst als unzuverlässige Instanz in Hinblick auf die eingangs vorgenommene Bewertung

Sie mich ohne Zaudern. Nein, ich bin keine Christin, ich bin eine Götzendienerin.“ (Ibid., S. 238)

${ }^{46}$ Menhard hält fest: „das Wirkungspotential von Multiperspektivität und unreliable narration im Text [wird] sowohl von der Frage nach der internen reliability einer Perspektive als auch von der Interaktion zwischen allen vorhandenen Perspektiven geprägt“ (MENHARD, Multiperspektivität, S. 4).

47 Vgl. dazu NüNNING, ,Einleitung“, S. 9: „Daher hat sich die Forschung zum Problem fiktionaler unreliability bislang fast ausschließlich auf den Typus des [...] Ich-Erzählers beschränkt [...]. Im Gegensatz dazu gelten auktoriale Erzähler [...] von vorneherein als vertrauenswürdig.“

48 Valera, Pepita Jiménez, S. 318. Dt.: „Ich erklärte gleich zu Anfang, daß ich vermute, der erzählende Teil, der den Titel ,Paralipomena' trägt, sei das Werk der Herrn Dekans gewesen [...]. Jetzt, da ich wahrnehme, mit welchem Freimut gewisse Stellen behandelt sind und welch weites Gewissen der Verfasser für manche Entgleisung des Priesterschülers zeigt, bin ich wieder schwankend geworden, ob die Niederschrift aus dem Tintenfaß des Herrn Dekans stammt. Zudem weiß ich aus guter Quelle, welch strenge Maßstäbe der geistliche Herr anzulegen pflegte“ (VAlera, Pepita Jiménez. Roman, S. 251f.). 
der ergänzenden Bemerkungen als „Werk des Herrn Dekans“. Die Freizügigkeit des Berichts etwa über das Tête-à-Tête der jungen Liebenden scheinen bei näherer Betrachtung mit den Überzeugungen eines Seminarrektors in der Tat nur schwer vereinbar. Die Frage nach der Identität des Paralipomena-Erzählers ist von der Valera-Forschung bereits aufgeworfen, nicht aber abschließend beantwortet worden, wobei sich manche Autoren dem Problem mit der Behauptung, es handele sich um eine „non-question“ schlicht entzogen haben. ${ }^{49}$ Ruano de La Haza hat zu belegen versucht, dass Don Pedro, der Vater des Protagonisten, als Erzähler der Paralipomena ungleich plausibler erscheint als der Dechant, habe er doch beispielsweise im Unterschied zum Dechanten Zutritt zu Pepitas Haus gehabt und gleiche seine Wahrnehmung der jungen Frau in den „Cartas de mi hermano“ manchen Passagen der Paralipomena. ${ }^{50}$ Doch so eindeutig wie Ruano de la Haza behauptet, lässt sich die Identität des Erzählers keineswegs bestimmen. Es findet sich nämlich im Text mindestens eine Passage, in der sich die Erzählerstimme des fiktiven Herausgebers mit der des Paralipomena-Erzählers vermischt, als nämlich der Herausgeber von einem editorialen Kommentar übergeht zu einem narrativen Rückblick ${ }^{51}$, mit dem die Erzählung fortgesetzt wird. Indem der Herausgeber an dieser Stelle als Erzählinstanz der Paralipomena fungiert, wirft der Text die Frage auf, ob nicht der Herausgeber selbst Urheber dieses Textes und ihr eigentlicher Erzähler ist, also auch die zitierten Einlassungen über die Autorschaft/ Nicht-Autorschaft des Dechanten (s.o.) nicht mehr gewesen sind als ein Täuschungsversuch, eine Lüge des Herausgebers.

\section{Fazit}

Nichts ist gewiss in Pepita Jiménez, kein Erzähler ist tatsächlich vollkommen zuverlässig, der Leser permanenter Zeuge eines Spiels des Romans mit der Kategorie der narrativen Wahrheit. In diesem ironischen, selbstreflexiven Spiel liegt das Ziel unverlässlichen Erzählens in Pepita Jiménez. ${ }^{52}$ Mit ihm knüpft Valera an die Tradition des cervantinischen Realismus und dessen metafiktionale Verfahren ${ }^{53}$ und unverlässliche Erzähler an: Unweigerlich muss man im Kontext der unreliable narration an Cide Hamete Benengeli aus dem Don Quijote denken. ${ }^{54}$ Alle Figuren

49 Vgl. James Whiston, Valera: Pepita Jiménez, London, Grant \& Cutler [u.a.], 1977.

50 Vgl. José M. Ruano de HazA, „La identidad del narrador de los Paralipómenos de Pepita Jiménez“, in Revista Canadiense de Estudios Hispánicos, VIII (3), 1984, S. 335-350.

51 Valera, Pepita Jiménez, S. 295-296.

52 Vgl. zur Bedeutung der Ironie in Pepita Jiménez den sehr kurzen, aber inspirierenden Aufsatz von Frank DuRAND, „Valera. Narrador irónico“, in Insula, 31 (360), 1976, S. 3. Orejas bezeichnet den Roman gar als „obra plenamente metafictiva“, ohne diese Einschätzung aber detaillierter zu begründen. Vgl. Francisco G. OREJAS, La metaficción en la novela española contemporánea. Entre 1975 y fin de siglo, Madrid, ARCO/ LIBROS, 2003, S. 233.

${ }^{53}$ Vgl. in Hinblick auf den Begriff der Metafiktion die Definition von Waugh: ,Metafiction is a term given to fictional writing which self-consciously and systematically draws attention to its status as an artefact in order to pose questions about the relationship between fiction and reality“ (Patricia WAUGH, Metafiction: The Theory and Pratice of Self-Conscious Fiction, London, Methuen, 1984, S. 2).

${ }^{54} \mathrm{Vgl}$. zu den strukturellen und sprachlichen Parallelen zwischen Pepita Jiménez und Don Quijote Robert E. LotT, Siglo de oro tradition and modern adolescent psychology in Pepita Jiménez. A stylistic 
des Romans sind in der ein oder anderen Weise als Autoren tätig: Luis und Pedro de Vargas als Briefeschreiber, der Herausgeber als Co-Autor des Romans, der Dechant als Verfasser der Paralipomena. Vermittels des unverlässlichen Erzählens dieser Figuren macht Valera die Bedingungen literarischen Schreibens sichtbar: Jeder narrative Text ist als Erzählung Produkt der Selektion, Komposition und Formung kurz: der künstlerischen Arbeit - eines Autors. Dieser allein trägt die Verantwortung für den Text, weder kann er neutraler Berichterstatter einer beobachteten ,Realität‘, noch bloßer Lieferant von ,Dokumenten“ sein. Dergestalt nimmt Valera mit Pepita Jiménez eine ästhetische Gegenposition zum französischen Realismus und Naturalismus und dessen Postulat quasi-naturwissenschaftlicher, objektiver Beobachtung ein, das er von seiner frühen Schaffensphase an in zahllosen Aufsätzen als ,ciencia y $[\ldots]$ no arte“ verdammte. ${ }^{55}$

study. Nachdr. der Ausg. Washington 1958, New York, AMS Press, 1976.

55 „Y aquí llamo ciencia al arte de escribir novelas naturalistas, porque ahora sacamos en claro que la tal novelería es ciencia y no es arte; y es ciencia, no así como se quiera, sino ciencia experimental“ (Juan VAlera, „Apuntes sobre el nuevo arte de escribir novelas“, in Obras completas, Bd. II, Madrid, Aguilar, 1949, S. 625). Dt.: „Die Kunst naturalistische Romane zu schreiben, nenne ich Wissenschaft, denn es ist klar, dass diese Romanschreiberei eine Wissenschaft ist und keine Kunst; und es ist nicht irgendeine Wissenschaft, sondern eine experimentelle Wissenschaft" [Übersetzung von mir]. Vgl. zur Bedeutung der Apuntes sobre el nuevo arte de escribir novelas Luis LóPEz JiméNEz, El naturalismo y España. Valera frente a Zola, Madrid, Alhambra, 1977. 